\title{
Study of HIV Disease and Its Association with Immune Cells under Nonsingular and Nonlocal Fractal-Fractional Operator
}

\author{
Shabir Ahmad $\left(\mathbb{D},{ }^{1}\right.$ Aman Ullah $\mathbb{D}^{1},{ }^{1}$ Ali Akgül $\mathbb{D}^{\circ},{ }^{2}$ and Manuel De la Sen $\mathbb{D D}^{3}$ \\ ${ }^{1}$ Department of Mathematics, University of Malakand, Dir (L), Khyber Pakhtunkhwa, Pakistan \\ ${ }^{2}$ Art and Science Faculty, Department of Mathematics, Siirt University, TR-56100 Siirt, Turkey \\ ${ }^{3}$ Institute of Research and Development of Processes Faculty of Science and Technology, University of the Basque Country, \\ Campus of Leioa (Bizkaia), 644-Leioa, Biscay, Spain \\ Correspondence should be addressed to Shabir Ahmad; shabirahmad2232@gmail.com
}

Received 18 May 2021; Revised 3 August 2021; Accepted 10 August 2021; Published 20 August 2021

Academic Editor: Ning Cai

Copyright (C) 2021 Shabir Ahmad et al. This is an open access article distributed under the Creative Commons Attribution License, which permits unrestricted use, distribution, and reproduction in any medium, provided the original work is properly cited.

\begin{abstract}
HIV, like many other infections, is a severe and lethal infection. Fractal-fractional operators are frequently used in modeling numerous physical processes in the current decade. These operators provide better dynamics of a mathematical model because these are the generalization of integer and fractional-order operators. This paper aims to study the dynamics of the HIV model during primary infection by fractal-fractional Atangana-Baleanu (AB) operators. The sufficient conditions for the existence and uniqueness of the solution of the proposed model under the AB operator are derived via fixed point theory. The numerical scheme is presented by using the Adams-Bashforth method. Numerical results are demonstrated for different fractal and fractional orders to see the effect of fractional order and fractal dimension on the dynamics of HIV and CD4+ T-cells during primary infection.
\end{abstract}

\section{Introduction}

Over the past few decades, the field of mathematical modeling of the physical process has gained considerable attention from scientists and investigators. We point out that mathematical models are important tools for studying many physical and biological science dynamic problems [1, 2]. Bernoulli has initiated this concept in 1776. Mathematical models of the biological problem have become important means for understanding the many infectious diseases and choosing the appropriate technique for controlling the disease or reducing its social transmission. In this respect, many mathematical models were built to study the understanding of many infectious diseases and to follow certain precautions to save a community from excessive loss. HIV is one of the most severe and dangerous illnesses of the last decades. Many people all over the world have died because of the disease. According to UNAIDS, about 690,000 people died from AIDS-related illnesses in 2019. The aforementioned infections target the largest WBCs in the immune system (IS) during HIV disease, called CD4 + Tcells [3]. In this way, HIV infection affects human IS. It has detrimental impacts on the CD4 + T-cells and other cells. A body becomes susceptible to diseases because the number of $\mathrm{CD} 4+\mathrm{T}$-cells falls below the amount needed, and thus the IS begins to weaken. Several countries, particularly in Africa, have recently been infected with HIV for up to $35 \%$ of the population aged $15-50$ years. Usually, differential equations are commonly used during mathematical models. A basic model for primary HIV infection was first developed in 1989 by Perelson [4]. In 1993, Perelson et al. [5] expanded the model and further addressed some of the behavior of the models. For examination of the HIV infection, the two cells model was established in [6]. A simple general model in [7] was considered. In this regard, numerous models have been further developed and to explain the dynamics of HIV decay observed and studied for local and global stability $[8,9]$. Further, Arafa et al. [10] have proposed the following model: 


$$
\left\{\begin{array}{l}
\frac{\mathrm{d} X}{\mathrm{~d} t}=\mu-k^{*} X Z-d^{*} X+b^{*} Y, \\
\frac{\mathrm{d} Y}{\mathrm{~d} t}=k^{*} X Z-\left(b^{*}+\delta\right) Y, \\
\frac{\mathrm{d} Z}{\mathrm{~d} t}=N^{*} \delta Y-c^{*} Z .
\end{array}\right.
$$

The symbol $X$ denotes the concentration of susceptible $\mathrm{CD}^{+}$T-cells, $Y$ represents concentration of infected CD4 + T-cells, and free HIV virus particles in the blood cells are denoted by $Z$. The parameter $\mu$ represents new T-cells supply rate. The rate of natural death is denoted by $d^{*}, k^{*}$ is the rate of infection T-cell, $\delta$ is the death rate of infected T-cells, $b^{*}$ represents the rate of return of infected cells to uninfected class, $c^{*}$ is the death rate of virus, and $N^{*}$ is average number of particles infected by an uninfected cell.

Fractional calculus theory has been a hot topic of the twenty-first century due to its applications in various fields of science. Different fractional operators have been implemented in many mathematical models, and they have yielded a lot of success. Different forms of operators exist in fractional calculus, depending on the kernels involved. The three major operators, which are widely used by researchers, are briefly discussed here. The Caputo operator, which is built on the power law kernel, is the first. This operator has a problem with the singularity of the kernel involved in it. The second operator is the Caputo-Fabrizio fractional derivative, which is based on an exponential-decay kernel but has a locality problem. The third operator is the $\mathrm{AB}$ fractional derivative which is based on the Mittag-Leffler kernel. Because of its nonlocal and nonsingular kernel, this derivative gives superior results as compared with Caputo and Caputo-Fabrizio. The FDEs had a significant impact on modeling and simulation using these three types of kernels $[11,12]$. In literature [1], computational solutions of the HIV-1 infection of the CD4+ T-cells fractional mathematical model that causes acquired immunodeficiency syndrome (AIDS) with the effect of antiviral drug therapy are presented. Khater et al. analyzed abundant stable computational solutions of Atangana-Baleanu fractional nonlinear HIV-1 infection of CD4+ T-cells of immunodeficiency syndrome [2]. In comparison to the classical model, the model involving FDEs is more accurate [13]. Various methods are used by researchers for solving linear and nonlinear fractional DEs $[14,15]$. Many problems in nature are solved by the concept of fractal derivatives. Atangana [16] recently proposed new kinds of general operators called fractalfractional operators, which combine fractional and fractal derivatives. The newly proposed operators have been implemented by many scientists to investigate the dynamics of different models. Ahmad et al. studied the model describing the tumor and its relation with immune cells under the $A B$ fractal-fractional operators [17]. Literature [18] has demonstrated the dynamics of the dengue infection model via fractal-fractional operators which are best fitted with real data. For more applications of fractional-fractal calculus, see
$[19,20]$. We will investigate the above model using the AB fractal-fractional operator, as suggested by the literature. We extend the above model as follows:

$$
\left\{\begin{array}{l}
\mathscr{D}_{0, t}^{\alpha, \tau} X=\mu-k^{*} X Z-d^{*} X+b^{*} Y, \\
\mathscr{D}_{0, t}^{\alpha, \tau} Y=k^{*} X Z-\left(b^{*}+\delta\right) Y, \\
\mathscr{D}_{0, t}^{\alpha, \tau} Z=N^{*} \delta Y-c^{*} Z,
\end{array}\right.
$$

along with the following initial conditions:

$$
\begin{aligned}
& X(0)=X_{0}, \\
& Y(0)=Y_{0}, \\
& Z(0)=Z_{0} .
\end{aligned}
$$

Here, we use more generalized operators to model HIV infections and their association with immune cells. We explore the existence theory under $A B$ fractal-fractional derivative through fixed point theory. Our proposed model is nonlinear. One common obstacle is determining the exact solution to a nonlinear problem. Due to the complexity, we will find a numerical solution to the model. The Adam Bashforth methodology is an efficient and stable numerical method. We use this method to determine the solution to the given model. We obtain the numerical scheme for the Mittag-Leffler law via the Adams-Bashforth technique. To present the effect of fractal dimension on fractional order, we simulate the proposed model for various values of fractal values. We find out that the $\mathrm{AB}$ operator provides better dynamics of the disease due to nonsingular kernel. We show the impact of fractal and fractional order on the dynamics of HIV infection and its association with immune cells.

The paper is structured as follows. The introduction and motivation part is presented in Section 1. The basic definitions of $\mathrm{AB}$ fractal-fractional operators are given in Section 2. The existence theory under AB fractal-fractional derivative is explored in Section 3. Section 4 is devoted to the numerical scheme for the proposed model. Numerical results are simulated in Section 5, whereas the manuscript is concluded in Section 6.

\section{Preliminaries}

Let $\mathscr{G}(t)$ be continuous and fractal differentiable on $(m, n)$. Let $0 \leq \alpha, \tau \leq 1$, where $\alpha$ and $\tau$ represent fractional and fractal order, respectively.

Definition 1 (see [16]). The AB fractal-fractional derivative of $\mathscr{G}(t)$ is defined as follows:

$$
\mathscr{F} \mathscr{F} \mathscr{M} \mathscr{D}_{0, t}^{\alpha, \tau}(\mathscr{G}(t))=\frac{\mathscr{A} \mathscr{B}(\alpha)}{1-\alpha} \frac{\mathrm{d}}{\mathrm{d} t^{\tau}} \int_{0}^{t} E_{\alpha}\left[-\frac{\alpha}{1-\alpha}(t-\xi)^{\alpha}\right] \mathscr{G}(\xi) \mathrm{d} \xi,
$$

where $\mathscr{A} \mathscr{B}(\alpha)=1-\alpha+(\alpha / \Gamma(\alpha))$.

Definition 2 (see [16]). The AB fractal-fractional integral of $\mathscr{G}(t)$ is defined as follows: 


$$
\mathscr{F} \mathscr{F} \mathscr{J}_{0, t}^{\alpha, \tau} \mathscr{G}(t)=\frac{\tau(1-\alpha) t^{\tau-1} \mathscr{G}(t)}{\mathscr{A} \mathscr{B}(\alpha)}+\frac{\alpha \tau}{\mathscr{A} \mathscr{B}(\alpha)} \int_{0}^{t} \xi^{\alpha-1}(t-\xi)^{\alpha-1} \mathscr{G}(\xi) \mathrm{d} \xi
$$

\section{Existence and Uniqueness}

In this section, we present the existence and uniqueness of the solution of the proposed model under $\mathrm{AB}$ fractal-fractional derivative via fixed point theory.

$$
\left\{\mathscr{F} \mathscr{F} \mathscr{M}_{\mathscr{D}_{0, t}^{\alpha, \tau}}^{\alpha}(\mathscr{X}(t))=\mathscr{H}_{1}(t, \mathscr{X}, Y, Z),{ }^{\mathscr{F} \mathscr{F}} \mathscr{D}_{0, t}^{\alpha, \tau}(Y(t))=\mathscr{H}_{2}(t, \mathscr{X}, Y, Z),{ }^{\mathscr{F} \mathscr{M}} \mathscr{D}_{0, t}^{\alpha, \tau}(Z(t))=\mathscr{H}{ }_{3}(t, \mathscr{X}, Y, Z),\right.
$$

where

$$
\left\{\begin{array}{l}
\mathscr{H}_{1}(\xi, \mathscr{X}, Y, Z)=\mu-k^{*} X Z-d^{*} X+b^{*} Y, \\
\mathscr{H}_{2}(\xi, \mathscr{X}, Y, Z)=k^{*} X Z-\left(b^{*}+\delta\right) Y, \\
\mathscr{H}_{3}(\xi, \mathscr{X}, Y, Z)=N^{*} \delta Y-c^{*} Z .
\end{array}\right.
$$

For this, we can write system (6) as follows:

$$
\mathscr{F} \mathscr{F} \mathscr{M}_{0, t}^{\alpha, \tau}[\Omega(t)]=\Xi(t, \Omega(t)),
$$

$$
\begin{aligned}
& \text { where } \Omega(t)=\left\{\begin{array}{l}
\mathscr{X}(t) \\
Y(t) \text { and } \Xi(t, \Omega(t))=\left\{\begin{array}{l}
\mathscr{H}_{1}(t, \mathscr{X}, Y, Z) \\
\mathscr{H}_{2}(t, \mathscr{X}, Y, Z) \\
\mathscr{H}_{3}(t) \\
\text { by }(t, \mathscr{X}, Y, Z)
\end{array}\right. \\
\quad \text { Since definition } \\
=(\mathscr{A} \mathscr{B}(\alpha) / 1-\alpha) \mathrm{d} / \mathrm{d} t^{\tau} \int_{0}^{t} \Xi(\vartheta, \Omega(\vartheta)) E_{\alpha}(-\alpha / 1
\end{array}\right. \\
& \left.-\alpha(t-\xi)^{\alpha}\right) \mathrm{d} \xi \text { and integral is differentiable, we can write the } \\
& \text { above expression as follows: }
\end{aligned}
$$

$$
\mathscr{F} \mathscr{F} \mathscr{M}_{0, t}^{\alpha}[\Omega(t)]=\frac{1}{\tau t^{\tau-1}} \frac{\mathscr{A} \mathscr{B}(\alpha)}{1-\alpha} \frac{\mathrm{d}}{\mathrm{d} t} \int_{0}^{t} \Xi(\vartheta, \Omega(\vartheta)) E_{\alpha}\left(-\frac{\alpha}{1-\alpha}(t-\xi)^{\alpha}\right) \mathrm{d} \xi .
$$

Therefore, system (8) can be expressed as follows:

$$
\frac{\mathscr{A} \mathscr{B}(\alpha)}{1-\alpha} \frac{\mathrm{d}}{\mathrm{d} t} \int_{0}^{t} \Xi(\vartheta, \Omega(\vartheta)) E_{\alpha}\left(-\frac{\alpha}{1-\alpha}(t-\xi)^{\alpha}\right) \mathrm{d} \xi=\tau t^{\tau-1} \Xi(t, \Omega(t)) .
$$

Substituting the right hand side by Caputo and implementing the fractional integral, we get

$$
\Omega(t)=\Omega(0)+\frac{1-\alpha}{\mathscr{A} \mathscr{B}(\alpha)} \tau t^{\tau-1} \Xi(t, \Omega(t))+\frac{\alpha \tau}{\mathscr{A} \mathscr{B}(\alpha) \Omega(\alpha)} \int_{0}^{t}(t-\xi)^{\alpha-1} \Xi(\xi, \Omega(\xi)) \xi^{\tau-1} \mathrm{~d} \xi
$$

Here, also like Picard Lindlof theorem, we let

$$
\prod_{a}^{b}=\mathscr{I}_{n}\left(t_{n}\right) \times \overline{\mathscr{A}_{0}\left(\Omega_{0}\right)}
$$

where $\mathscr{I}_{n}\left(t_{n}\right)=\left[t_{n-a}, t_{n+a}\right]$ and $\overline{\mathscr{A}_{0}\left(\Omega_{0}\right)}=\left[t_{0}-b, t_{0}+b\right]$. Also, let $\sup _{t \in \prod^{b}}\|\Xi\|=\mathscr{K}$; now, we define the norm as follows: 


$$
\|\Psi\|_{\infty}=\sup _{t \in \prod_{a}^{b}|\Psi(t)| .}
$$

Next, we define the operator O: $\mathscr{C}\left[\mathscr{I}_{n}\left(t_{n}\right), \mathscr{A}_{b}\left(t_{n}\right)\right] \longrightarrow \mathscr{C}\left[\mathscr{I}_{n}(b), \mathscr{A}_{b}\left(t_{n}\right)\right]$ as

$$
\mathcal{O} \Omega(t)=\Omega_{0}+\frac{1-\alpha}{\mathscr{A} \mathscr{B}(\alpha)} \tau t^{\tau-1} \Xi(t, \Omega(t))+\frac{\alpha \tau}{\mathscr{A} \mathscr{B}(\alpha) \Gamma(\alpha)} \int_{0}^{t} \xi^{\tau-1}(t-\xi)^{\alpha-1} \Xi(\xi, \Omega(\xi)) \mathrm{d} \xi
$$

The goal is to show that the defined operator is a contraction mapping that translates a complete norm empty metric space $\mathrm{Y}$ into itself. First, we need to show that $\left\|\mathcal{O} \Omega(t)-\Omega_{0}\right\| \leq b$. For this, consider

$$
\begin{aligned}
& \left\|\mathscr{O} \Omega(t)-\Omega_{0}\right\| \leq \frac{1-\alpha}{\mathscr{A} \mathscr{B}(\alpha)} \tau t^{\xi-1}\|\Xi(t, \Omega(t))\|_{\infty} \\
& +\frac{\alpha \tau}{\mathscr{A} \mathscr{B}(\alpha) \Omega(\alpha)} \int_{0}^{t}(t-\xi)^{\alpha-1}\|\Xi(\xi, \Omega(\xi))\| \xi^{\tau-1} \mathrm{~d} \xi \\
& \leq \frac{1-\alpha}{\mathscr{A} \mathscr{B}(\alpha)} \tau t^{\tau-1} \mathscr{K}+\frac{\alpha \tau}{\mathscr{A} \mathscr{B}(\alpha) \Gamma(\alpha)} \mathscr{K} \int_{0}^{t}(t-\xi)^{\alpha-1} \xi^{\tau-1} \mathrm{~d} \xi .
\end{aligned}
$$

Let $\xi=t y$, expression (15) becomes

$$
\left\|\mathscr{O} \Omega(t)-\Omega_{0}\right\| \leq \frac{1-\alpha}{\mathscr{A} \mathscr{B}(\alpha)} \tau t^{\xi-1} \mathscr{K}+\frac{\alpha \tau \mathscr{K}}{\mathscr{A} \mathscr{B}(\alpha) \Gamma(\alpha)} t^{\alpha+\tau-1} \mathscr{B}(\alpha, \tau)
$$

where $\mathscr{B}(\alpha, \tau)$ represents beta function; thus,

$$
\left\|\mathcal{O} \Omega(t)-\Omega_{0}\right\| \leq b \longrightarrow \mathscr{K}<\frac{b \mathscr{B}(\alpha, \tau)}{\Gamma(\alpha)(1-\alpha) \tau t^{\tau-1}+\alpha \tau t^{\tau+\alpha-1} / \mathscr{A} \mathscr{B}(\alpha) \Gamma(\alpha)}
$$

Now, for any $\Omega_{1}, \Omega_{2} \in \mathscr{C}\left[\mathscr{I}_{n}\left(t_{n}\right), \mathscr{A}_{b}\left(t_{n}\right)\right]$, we have

$$
\begin{aligned}
& \left\|\mathcal{O} \Omega_{1}-\mathcal{O} \Omega_{2}\right\| \leq \frac{1-\alpha}{\mathscr{A} \mathscr{B}(\alpha)} \tau t^{\tau-1}\left\|\Xi\left(t, \Omega_{1}(t)\right)-\Xi\left(t, \Omega_{2}(t)\right)\right\| \\
& +\frac{\alpha \tau}{\mathscr{A} \mathscr{B}(\alpha) \Gamma(\alpha)} \int_{0}^{t}(t-\xi)^{\alpha-1} \xi^{\tau-1}\left\|\Xi\left(t, \Omega_{1}(t)\right)-\Xi\left(t, \Omega_{2}(t)\right)\right\| \mathrm{d} \xi
\end{aligned}
$$

Since $\Xi$ being a contraction, we have

$$
\begin{aligned}
\| \mathcal{O} & \Omega_{1}-\mathcal{O} \Omega_{2}\left\|\leq \frac{1-\alpha}{\mathscr{A} \mathscr{B}(\alpha)} \tau t^{\tau-1} \mathfrak{\Omega}\right\| \Omega_{1}-\Omega_{2} \|_{\infty} \\
& +\frac{\alpha \tau \mathfrak{Q}}{\mathscr{A} \mathscr{B}(\alpha) \Gamma(\alpha)}\left\|\Omega_{1}-\Omega_{2}\right\|_{\infty} \int_{0}^{t}(t-\xi)^{\alpha-1} \xi^{\tau-1} \mathrm{~d} \xi \leq \frac{1-\alpha}{\mathscr{A} \mathscr{B}(\alpha)} \tau t^{\tau-1} \mathfrak{\Omega}\left\|\Omega_{1}-\Omega_{2}\right\|_{\infty} \\
& +\frac{\alpha \tau \mathbb{Q}}{\mathscr{A} \mathscr{B}(\alpha) \Gamma(\alpha)}\left\|\Omega_{1}-\Omega_{2}\right\|_{\infty} t^{\alpha+\tau-1} \mathscr{B}(\alpha, \tau) .
\end{aligned}
$$

It follows that 


$$
\left\|\mathscr{O} \Omega_{1}-\mathscr{O} \Omega_{2}\right\| \leq\left[\frac{1-\alpha}{\mathscr{A} \mathscr{B}(\alpha)} \tau t^{\tau-1} \mathfrak{Q}+\frac{\alpha \tau \mathfrak{Q}}{\mathscr{A} \mathscr{B}(\alpha) \Gamma(\alpha)} t^{\alpha+\tau-1} \mathscr{B}(\alpha, \tau)\right]\left\|\Omega_{1}-\Omega_{2}\right\|_{\infty} .
$$

Now, since

$$
\begin{aligned}
& \frac{1-\alpha}{\mathscr{A} \mathscr{B}(\alpha)} \tau t^{\tau-1} \mathfrak{L}+\frac{\alpha \tau \mathfrak{Q}}{\mathscr{A} \mathscr{B}(\alpha) \Gamma(\alpha)} t^{\alpha+\tau-1} \mathscr{B}(\alpha, \tau) \\
& <\frac{1-\alpha}{\mathscr{A} \mathscr{B}(\alpha)} \tau a^{\tau-1} \mathfrak{Q}+\frac{\alpha \tau \mathfrak{Q}}{\mathscr{A} \mathscr{B}(\alpha) \Gamma(\alpha)} a^{\alpha+\tau-1} \mathscr{B}(\alpha, \tau) .
\end{aligned}
$$

Thus, $\mathcal{O}$ is a contraction if

$$
\left\|\mathcal{O} \Omega_{1}-\mathcal{O} \Omega_{2}\right\|_{\infty}<\left\|\Omega_{1}-\Omega_{2}\right\| .
$$

For this, we have

$\mathfrak{L}<\frac{1}{(1-\alpha / \mathscr{A} \mathscr{B}(\alpha)) \tau a^{\tau-1}+(\alpha \tau / \mathscr{A} \mathscr{B}(\alpha) \Gamma(\alpha)) a^{\alpha+\tau-1} \mathscr{B}(\alpha, \tau)}$, so

$$
\mathscr{K}<\frac{1}{(1-\alpha / \mathscr{A} \mathscr{B}(\alpha)) \tau a^{\tau-1}+(\alpha \tau / \mathscr{A} \mathscr{B}(\alpha) \Gamma(\alpha)) a^{\alpha+\tau-1} \mathscr{B}(\alpha, \tau)} .
$$

Thus, a unique solution of the proposed model exists.

\section{Numerical Scheme with Mittag-Leffler Type Kernel}

Consider model (2) as follows:

$$
\left\{{ }^{\mathscr{B} \mathscr{R}} \mathscr{D}_{0, t}^{\alpha}(\mathscr{X}(t))=\tau t^{\tau-1} \mathscr{H}_{1}(t, \mathscr{X}, Y, Z),{ }^{\mathscr{B} \mathscr{R}} \mathscr{D}_{0, t}^{\alpha}(Y(t))=\tau t^{\tau-1} \mathscr{H}_{2}(t, \mathscr{X}, Y, Z),{ }^{\mathscr{R} \mathscr{R}} \mathscr{D}_{0, t}^{\alpha}(Z(t))=\tau t^{\tau-1} \mathscr{H}{ }_{3}(t, \mathscr{X}, Y, Z) .\right.
$$

Then, we reach

$$
\left\{\begin{array}{l}
X(t)=\mathscr{X}(0)+\frac{\tau t^{\tau-1}(1-\alpha)}{\mathscr{A} \mathscr{B}(\alpha)} \mathscr{H}_{1}(t, \mathscr{X}, Y, Z) \\
+\frac{\alpha \tau}{\mathscr{A} \mathscr{B}(\alpha) \Gamma(\alpha)} \int_{0}^{t} \xi^{\tau-1}(t-\xi)^{\alpha-1} \mathscr{H}_{1}(\xi, \mathscr{X}, Y, Z) \mathrm{d} \xi, \\
Y(t)=Y(0)+\frac{\tau t^{\tau-1}(1-\alpha)}{\mathscr{A} \mathscr{B}(\alpha)} \mathscr{H}_{2}(t, \mathscr{X}, Y, Z) \\
+\frac{\alpha \tau}{\mathscr{A} \mathscr{B}(\alpha) \Gamma(\alpha)} \int_{0}^{t} \xi^{\tau-1}(t-\xi)^{\alpha-1} \mathscr{H}_{2}(\xi, \mathscr{X}, Y, Z) \mathrm{d} \xi, \\
\mathscr{E}(t)=Z(0)+\frac{\tau t^{\tau-1}(1-\alpha)}{\mathscr{A} \mathscr{B}(\alpha)} \mathscr{H}_{3}(t, \mathscr{X}, Y, Z) \\
+\frac{\alpha \tau}{\mathscr{A} \mathscr{B}(\alpha) \Gamma(\alpha)} \int_{0}^{t} \xi^{\tau-1}(t-\xi)^{\alpha-1} \mathscr{H}{ }_{3}(\xi, \mathscr{X}, Y, Z) \mathrm{d} \xi .
\end{array}\right.
$$

Now, at $t=t_{n+1}$, we have

$$
\left\{\begin{array}{l}
\mathscr{X}^{n+1}=\mathscr{X}^{0}+\frac{\tau t_{n}^{\tau-1}(1-\alpha)}{\mathscr{A} \mathscr{B}(\alpha)} \mathscr{H}_{1}\left(t_{n}, \mathscr{X}^{n}, Y^{n}, Z^{n}\right) \\
+\frac{\alpha \tau}{\mathscr{A} \mathscr{B}(\alpha) \Gamma(\alpha)} \int_{0}^{t_{n+1}} \xi^{\tau-1}\left(t_{n+1}-\xi\right)^{\alpha-1} \mathscr{H}_{1}(\xi, \mathscr{X}, Y, Z) \mathrm{d} \xi \\
\mathscr{Y}^{n+1}=\mathscr{Y}^{0}+\frac{\tau t_{n}^{\tau-1}(1-\alpha)}{\mathscr{A} \mathscr{B}(\alpha)} \mathscr{H}_{2}\left(t_{n}, \mathscr{X}^{n}, Y^{n}, Z^{n}\right) \\
+\frac{\alpha \tau}{\mathscr{A} \mathscr{B}(\alpha) \Gamma(\alpha)} \int_{0}^{t_{n+1}} \xi^{\tau-1}\left(t_{n+1}-\xi\right)^{\alpha-1} \mathscr{H}_{2}(\xi, \mathscr{X}, Y, Z) \mathrm{d} \xi \\
\mathscr{Z}^{n+1}=\mathscr{Z}^{0}+\frac{\tau t_{n}^{\tau-1}(1-\alpha)}{\mathscr{A}_{\mathscr{B}}(\alpha)} \mathscr{H}_{2}\left(t_{n}, \mathscr{X}^{n}, Y^{n}, Z^{n}\right) \\
+\frac{\alpha \tau}{\mathscr{A} \mathscr{B}(\alpha) \Gamma(\alpha)} \int_{0}^{t_{n+1}} \xi^{\tau-1}\left(t_{n+1}-\xi\right)^{\alpha-1} \mathscr{H}_{2}(\xi, \mathscr{X}, Y, Z) \mathrm{d} \xi .
\end{array}\right.
$$

Using the approximation of the integrals in (27), we get 
6

Complexity

$$
\left\{\begin{array}{l}
\mathscr{X}^{n+1}=\mathscr{X}^{0}+\frac{\tau t_{n}^{\tau-1}(1-\alpha)}{\mathscr{A} \mathscr{B}(\alpha)} \mathscr{H}_{1}\left(t_{n}, \mathscr{X}^{n}, Y^{n}, Z^{n}\right) \\
+\frac{\alpha \tau}{\mathscr{A} \mathscr{B}(\alpha) \Gamma(\alpha)} \sum_{f=0}^{p} \int_{t_{f}}^{t_{f+1}} \xi^{\tau-1}\left(t_{n+1}-\xi\right)^{\alpha-1} \mathscr{H}_{1}(\xi, \mathscr{X}, Y, Z) \mathrm{d} \xi \\
\mathscr{Y}^{n+1}=\mathscr{Y}^{0}+\frac{\tau t_{n}^{\tau-1}(1-\alpha)}{\mathscr{A} \mathscr{B}(\alpha)} \mathscr{H}_{2}\left(t_{n}, \mathscr{X}^{n}, Y^{n}, Z^{n}\right) \\
+\frac{\alpha \tau}{\mathscr{A} \mathscr{B}(\alpha) \Gamma(\alpha)} \sum_{f=0}^{p} \int_{t_{f}}^{t_{f+1}} \xi^{\tau-1}\left(t_{n+1}-\xi\right)^{\alpha-1} \mathscr{H}_{2}(\xi, \mathscr{X}, Y, Z) \mathrm{d} \xi \\
\mathscr{Z}^{n+1}=\mathscr{Z}^{0}+\frac{\tau t_{n}^{\tau-1}(1-\alpha)}{\mathscr{A} \mathscr{B}(\alpha)} \mathscr{H}_{2}\left(t_{n}, \mathscr{X}^{n}, Y^{n}, Z^{n}\right) \\
+\frac{\alpha \tau}{\mathscr{A} \mathscr{B}(\alpha) \Gamma(\alpha)} \sum_{f=0}^{p} \int_{t_{f}}^{t_{f+1}} \xi^{\tau-1}\left(t_{n+1}-\xi\right)^{\alpha-1} \mathscr{H}_{2}(\xi, \mathscr{X}, Y, Z) \mathrm{d} \xi
\end{array}\right.
$$

Now, utilizing the Lagrangian polynomial piecewise interpolation, one can get

$$
\left\{\begin{array}{l}
X^{n+1}=X^{0}+\frac{\tau t_{n}^{\tau-1}(1-\alpha)}{\mathscr{A} \mathscr{B}(\alpha)} \mathscr{H}_{1}\left(t_{n}, X^{n}, Y^{n}, Z^{n}\right)+\frac{\tau(\Delta t)^{\alpha}}{\mathscr{A} \mathscr{B}(\alpha) \Gamma(\alpha+2)} \\
\times \sum_{f=0}^{p}\left[\begin{array}{c}
t_{f}^{\tau-1} \mathscr{H}_{1}\left(t_{f}, X^{f}, Y^{f}, Z^{f}\right) \times\left((p+1-f)^{\alpha}(p-f+2+\alpha)-(p-f)^{\alpha}(p-f+2+2 \alpha)\right) \\
-t_{f-1}^{\tau-1} \mathscr{H}_{1}\left(t_{f-1}, X^{f-1}, Y^{f-1}, Z^{f-1}\right) \times\left((p-f+1)^{\alpha+1}-(p-f)^{\alpha}(p-f+1+\alpha)\right)
\end{array}\right] \\
Y^{n+1}=Y^{0}+\frac{\tau t_{n}^{\tau-1}(1-\alpha)}{\mathscr{A} \mathscr{B}(\alpha)} \mathscr{H}_{2}\left(t_{n}, X^{n}, Y^{n}, Z^{n}\right)+\frac{\tau(\Delta t)^{\alpha}}{\mathscr{A} \mathscr{B}(\alpha) \Gamma(\alpha+2)} \\
\times \sum_{f=0}^{p}\left[\begin{array}{c}
t_{f}^{\tau-1} \mathscr{H}_{1}\left(t_{f}, X^{f}, Y^{f}, Z^{f}\right) \times\left((p+1-f)^{\alpha}(p-f+2+\alpha)-(p-f)^{\alpha}(p-f+2+2 \alpha)\right) \\
-t_{f-1}^{\tau-1} \mathscr{H}_{1}\left(t_{f-1}, X^{f-1}, Y^{f-1}, Z^{f-1}\right) \times\left((p-f+1)^{\alpha+1}-(p-f)^{\alpha}(p-f+1+\alpha)\right)
\end{array}\right] \\
\times \sum_{f=0}^{p}\left[\begin{array}{c}
t_{f}^{\tau-1} \mathscr{H}_{1}\left(t_{f}, X^{f}, Y^{f}, Z^{f}\right) \times\left((p+1-f)^{\alpha}(p-f+2+\alpha)-(p-f)^{\alpha}(p-f+2+2 \alpha)\right) \\
-t_{f-1}^{\tau-1} \mathscr{H}_{1}\left(t_{f-1}, X^{f-1}, Y^{f-1}, Z^{f-1}\right) \times\left((p-f+1)^{\alpha+1}-(p-f)^{\alpha}(p-f+1+\alpha)\right)
\end{array}\right]
\end{array}\right.
$$




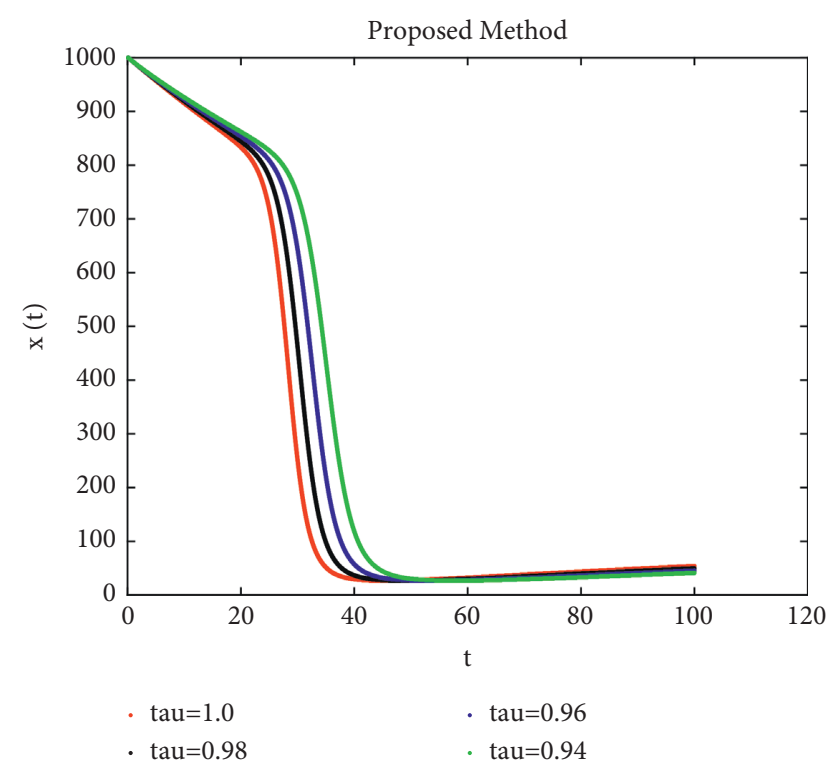

FIGURE 1: Dynamical behavior of concentration of uninfected (susceptible) cells at $\alpha=1$ and various fractal order under power law kernel.

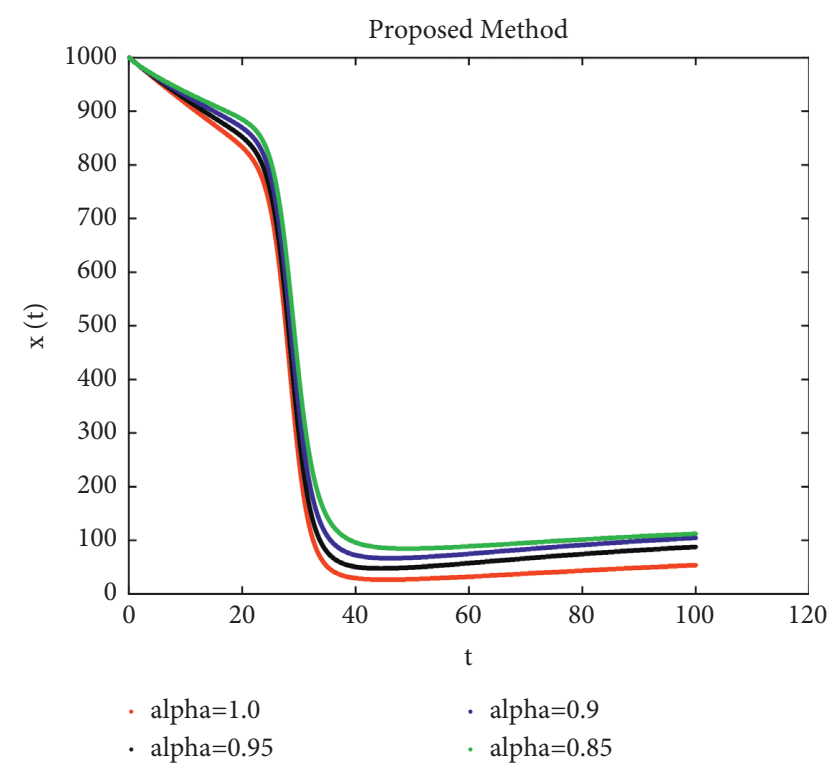

Figure 2: Dynamical behavior of concentration of uninfected (susceptible) cells at $\tau=1$ and various fractional order under power law kernel.

\section{Numerical Simulations}

In this section, we discuss the numerical simulations of the proposed model. For the desired simulation, we take $X_{0}=1000$ (millions), $Y_{0}=0$, and $Z_{0}=0.001$ (millions). The parameter values are as follows: $\mu=10, d^{*}=0.01$, $k^{*}=0.000024, \delta=0.16, b^{*}=0.02, c^{*}=3.4$, and $N^{*}=1000$. Via MATLAB, we graphically present the different

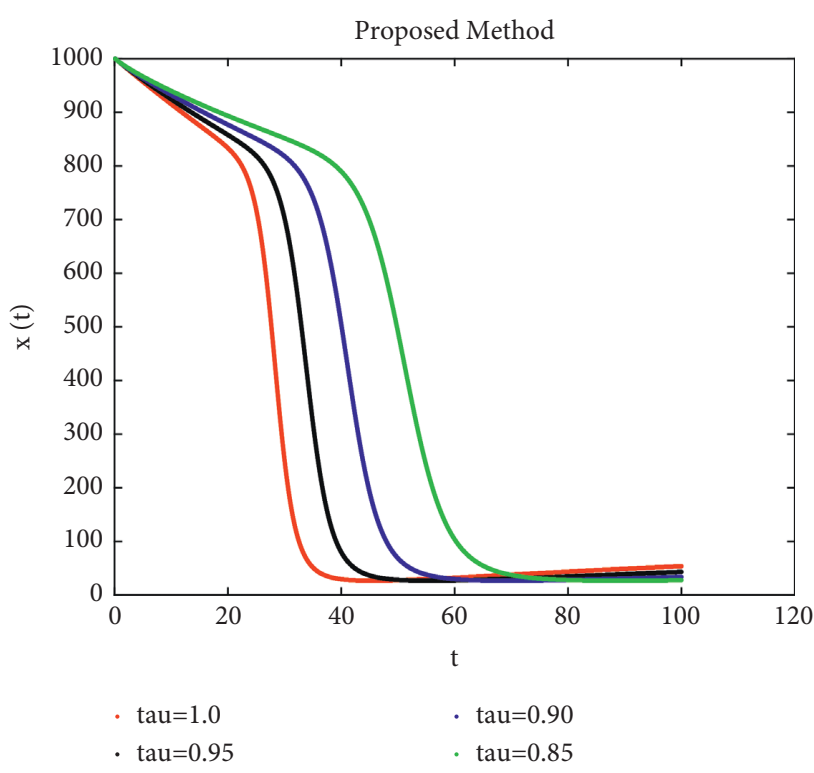

FIGURE 3: Dynamical behavior of concentration of uninfected (susceptible) cells at $\alpha=1$ and various fractal order $=0.85,0.9,0.95,1$ under power law kernel.

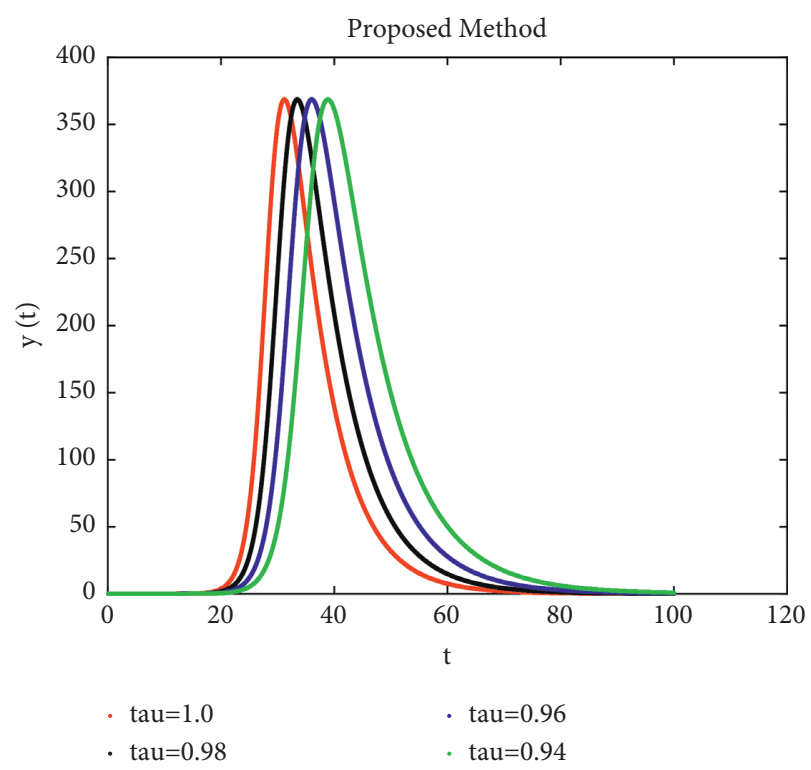

Figure 4: Dynamical behavior infected cells at $\alpha=1$ and fractal order under power law kernel.

compartments of the model against the various fractional and fractal orders. Figures 1-9 represent the dynamics of the proposed model for the Caputo fractal-fractional operator, and Figures 10-18 represent the dynamics suggested for the Atangana-Baleanu fractal-fractional operator. It is observed in Figures 1-3 and 10-12, which is the primary stage of HIV infection, the concentration level of uninfected CD4+ T-cells 


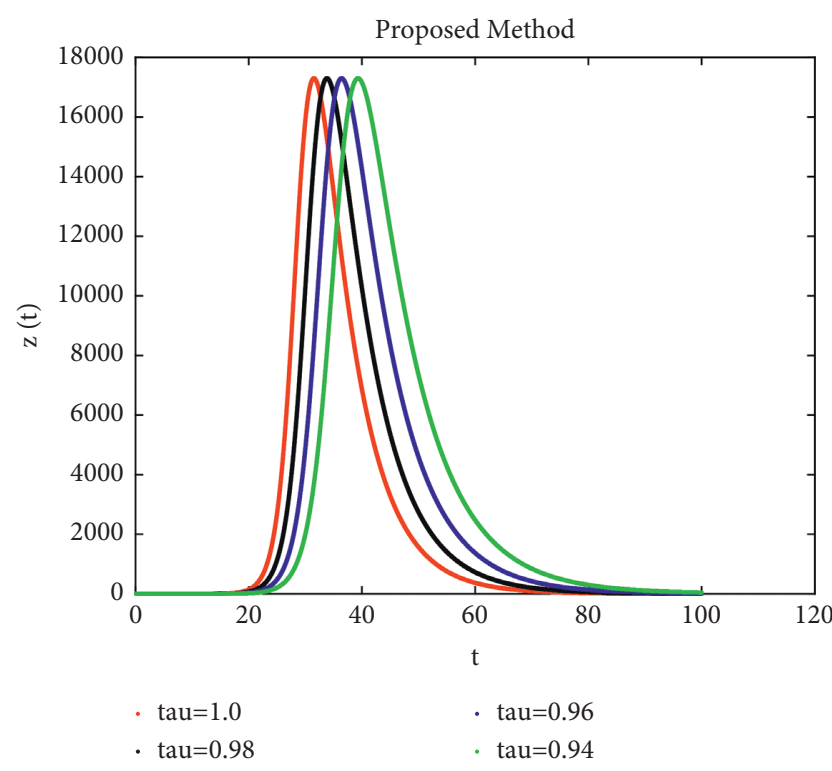

Figure 5: Dynamical behavior of free HIV virus particles at $\alpha=1$ and various fractal order under power law kernel.

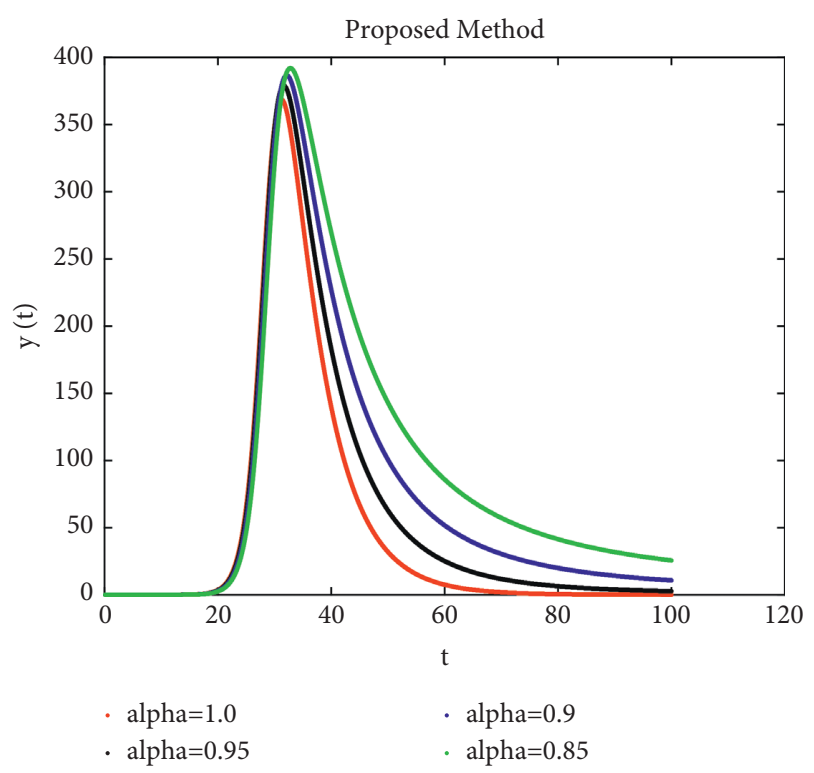

FIGURE 6: Dynamical behavior infected cells at $\tau=1$ and various fractional order under power law kernel.

is significantly decreased. It is because such infected cells have died. This decrease occurs mainly due to fractal and fractional order, with different rates. The smaller the order, the faster the decay, and thus the stability takes place before the highest order. On the other hand, we analyze from Figures 4-8 that the amount of infected CD4+ T-cells and free HIV particles is increasing. This rapid increase is also different in different fractal and fractional orders, and in smaller orders, it is faster, and as the order increases, the

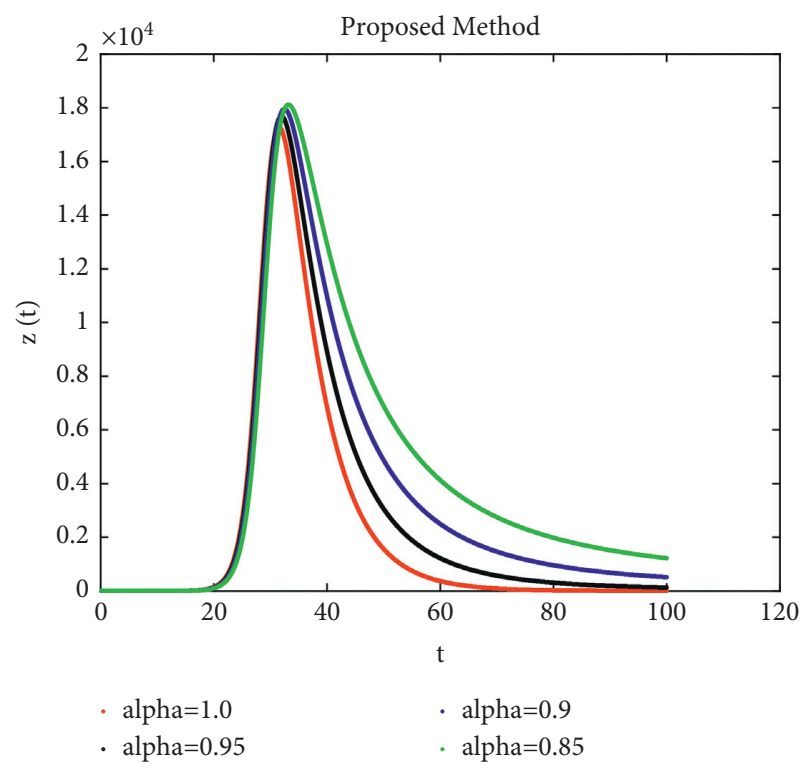

FIgURE 7: Dynamical behavior of free HIV virus particles at $\tau=1$ and various fractional order under power law kernel.

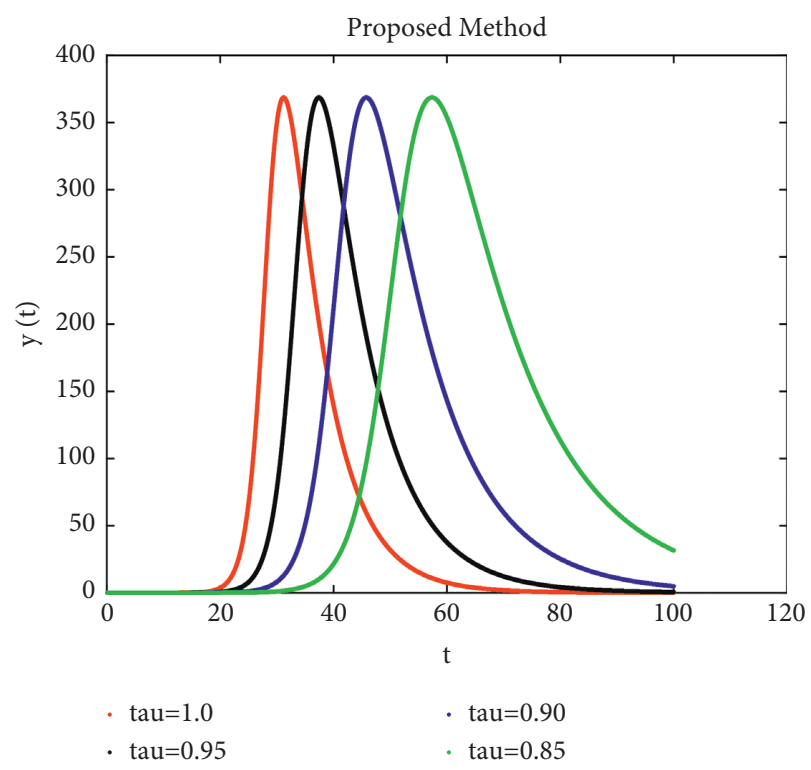

Figure 8: Dynamical behavior infected cells at $\alpha=1$ and various fractal order $=0.85,0.9,0.95,1$ under power law kernel.

growth rate becomes slower. From these graphic representations, it is clear that the development of healthy T-cells slows during HIV infection. We conclude from the figures that the use of fractal-fractional to solve an epidemic model gives the best results relative to fractional systems. We provide numerical simulations with different sets of fractal and fractional order for the proposed model. We observe the effect of the fractal dimension on the dynamics of the model. 


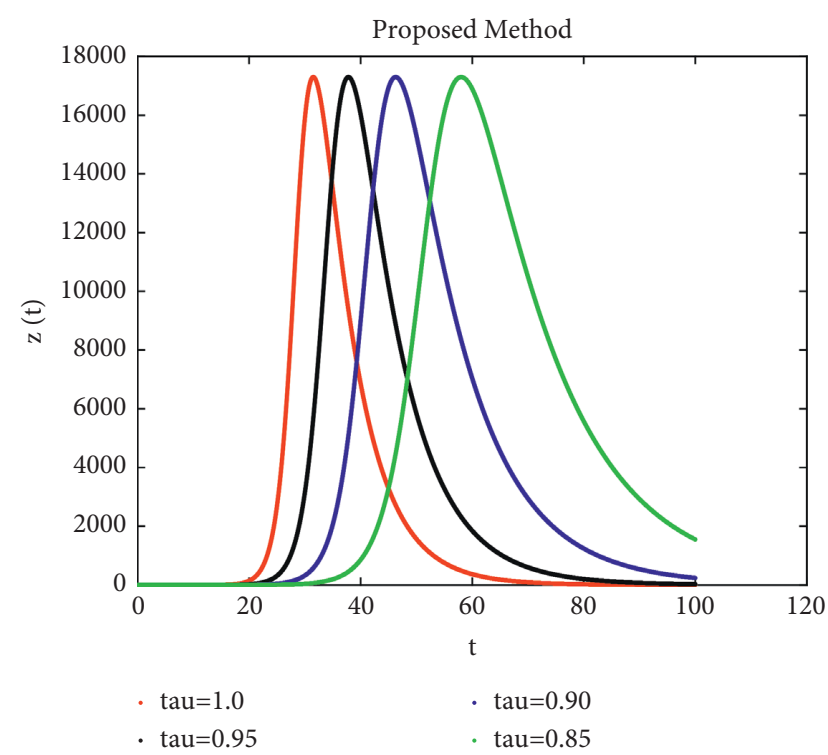

Figure 9: Dynamical behavior of free HIV virus particles at $\alpha=1$ and various fractal order $=0.85,0.9,0.95,1$ under power law kernel.

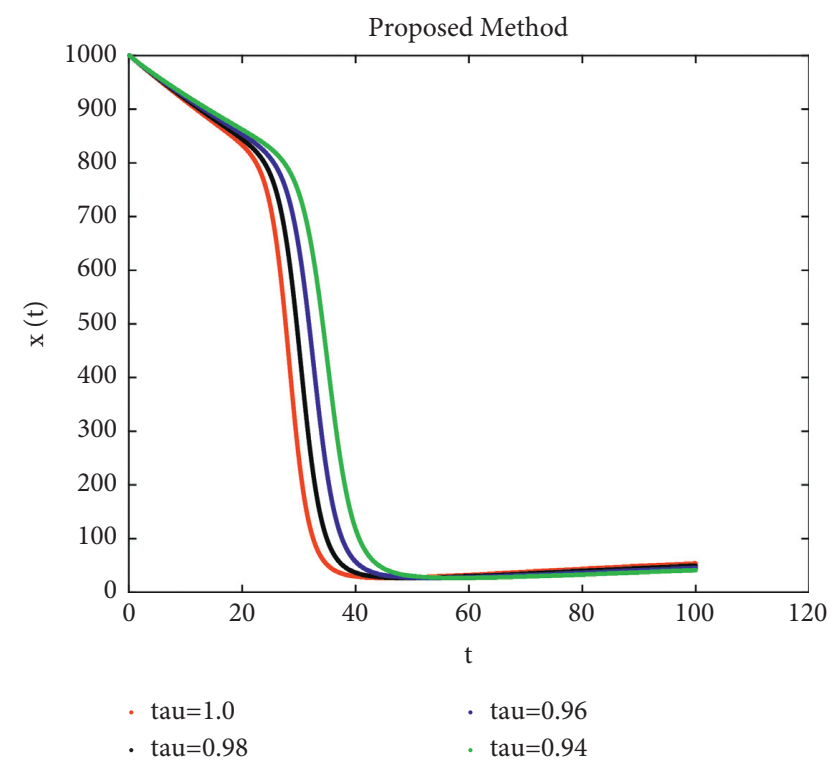

FIgURE 10: Dynamical behavior of concentration of uninfected (susceptible) cells at $\alpha=1$ and various fractal order under MittagLeffler kernel.

Also, we provide a comparison between Caputo and Atangana-Beleanu operators via numerical simulations. We have almost same result for the both operators for $\alpha=1$ and $\tau=0.85,0.9,0.95,1$ (see Figures 1, 4, 5, 10, 13, and 14). However, by changing fractional order and keeping fractal dimension fix, Atangana-Baleanu gives better dynamics of the proposed model. From Figures 2, 6, and 7, one can easily observe that there is no clear information about the population class for up to 40 days when changing the fractional

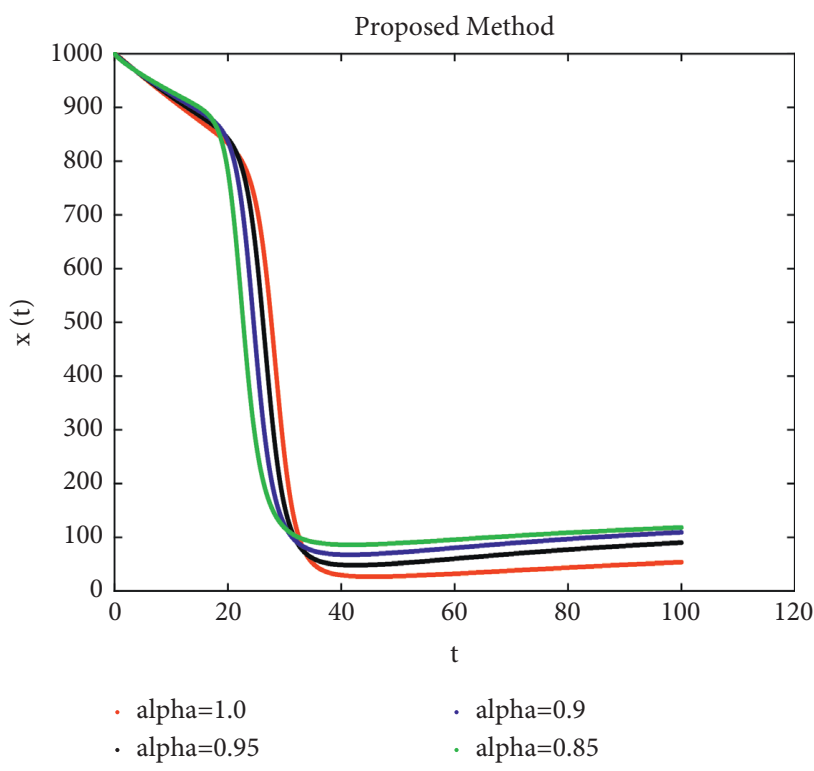

FIGURE 11: Dynamical behavior of concentration of uninfected (susceptible) cells at $\tau=1$ and various fractional order under Mittag-Leffler kernel.

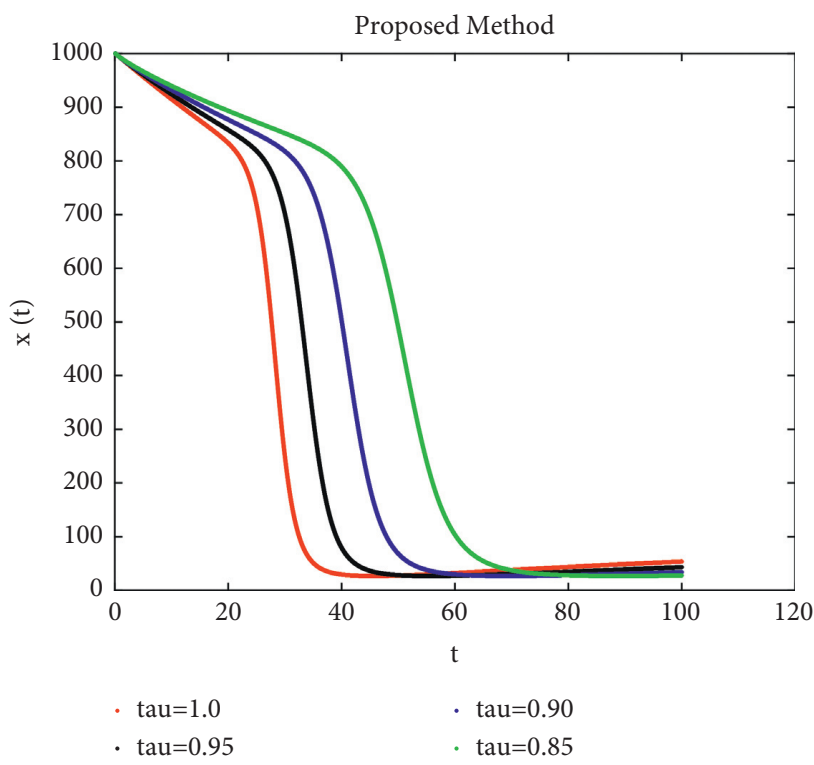

Figure 12: Dynamical behavior of concentration of uninfected (susceptible) cells at $\alpha=1$ and various fractal order $=0.85,0.9,0.95$, 1 under Mittag-Leffler kernel.

order. However, from Figures 11, 15, and 16, the effect of fractional order on the dynamics of the proposed model is much more clear. There is clear information of memory property about population class up to 40 days when changing the fractional order. It provides all previous history of the diseases, which is the main function of fractional order. Further, in Figure 19, we compare our simulated 


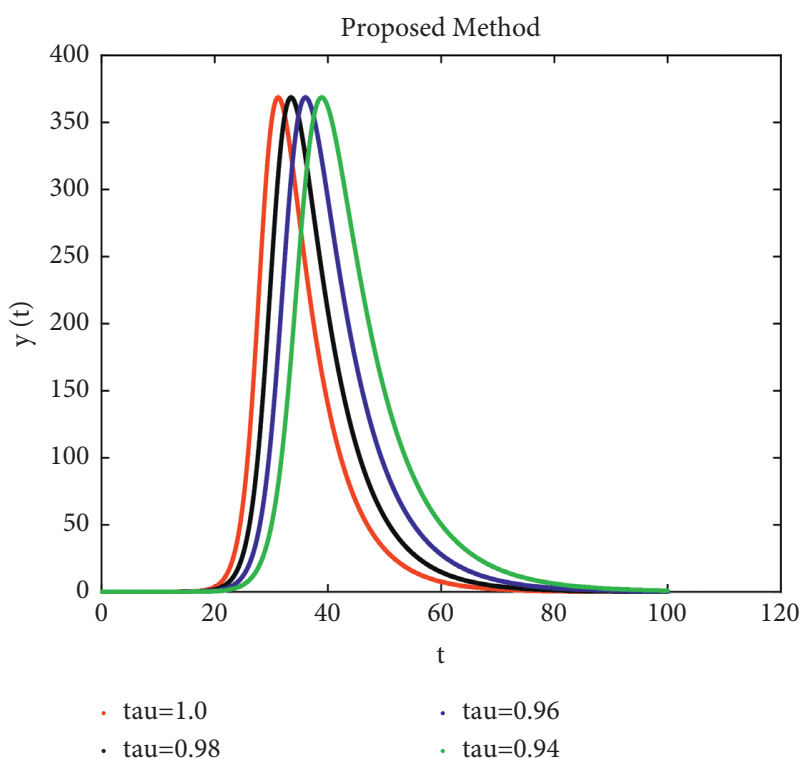

FIGURE 13: Dynamical behavior of infected cells at $\alpha=1$ and various fractal order under Mittag-Leffler kernel.

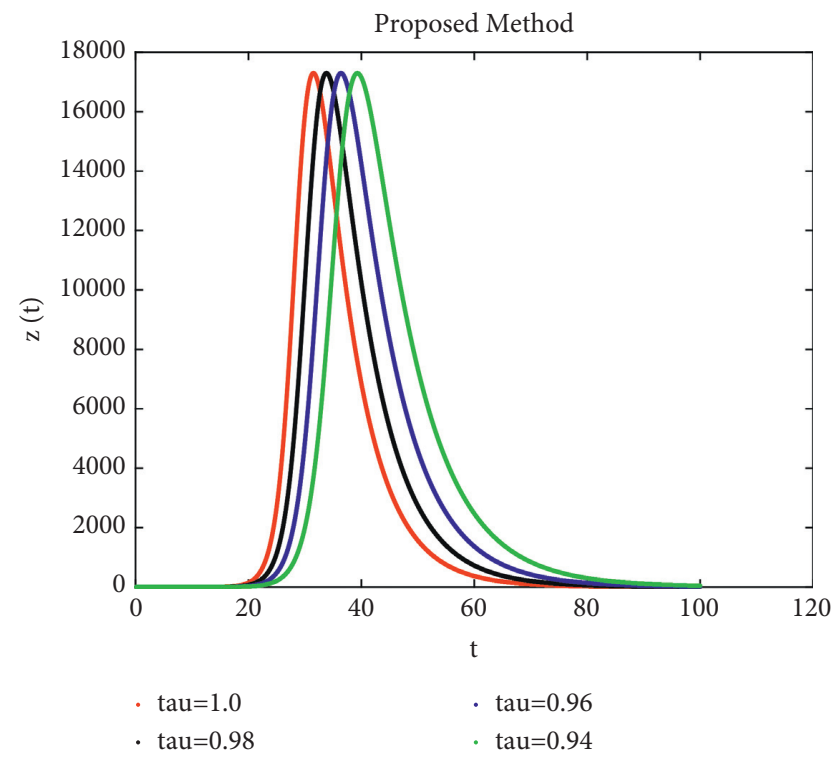

FIgure 14: Dynamical behavior of free HIV virus particles at $\alpha=1$ and various fractal order under Mittag-Leffler kernel.

results in the case of Atangana-Baleanu operator with the actual data for the country of Brazil. We have taken the data of HIV-infected people for 15 years from [21]. The red dots represent actual data, and the green curve represents the dynamics of infected HIV cells under the Atangana-Baleanu operator at fractional order 0.94 . We see that the graph of

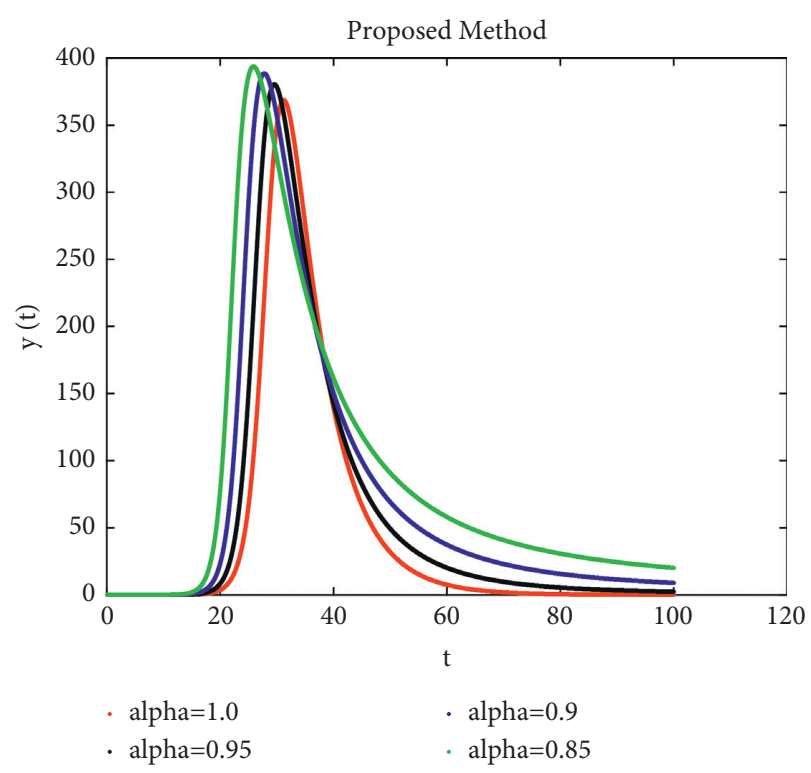

Figure 15: Dynamics of infected cells at $\tau=1$ and various fractional order under Mittag-Leffler kernel.

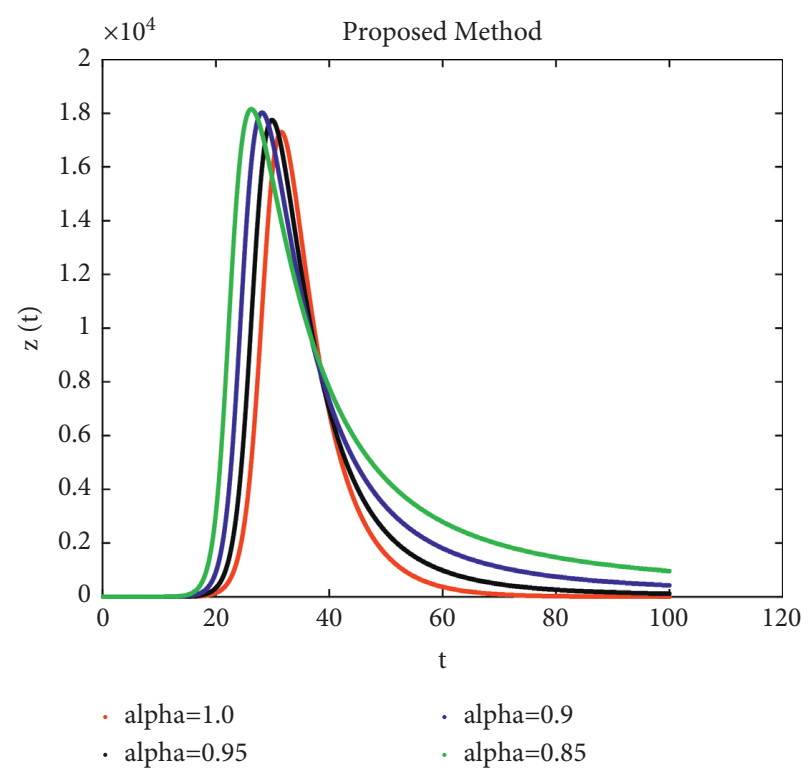

FIgURE 16: Dynamical behavior of free HIV virus particles at $\tau=1$ and various fractional order under Mittag-Leffler kernel.

simulated data and actual data is very close to each other at the order of 0.94 . On this basis, we conclude that fractalfractional operator in Atangana-Baleanu sense provides a clear understanding of an epidemic model's dynamics and can be applied successfully to several other problems. 


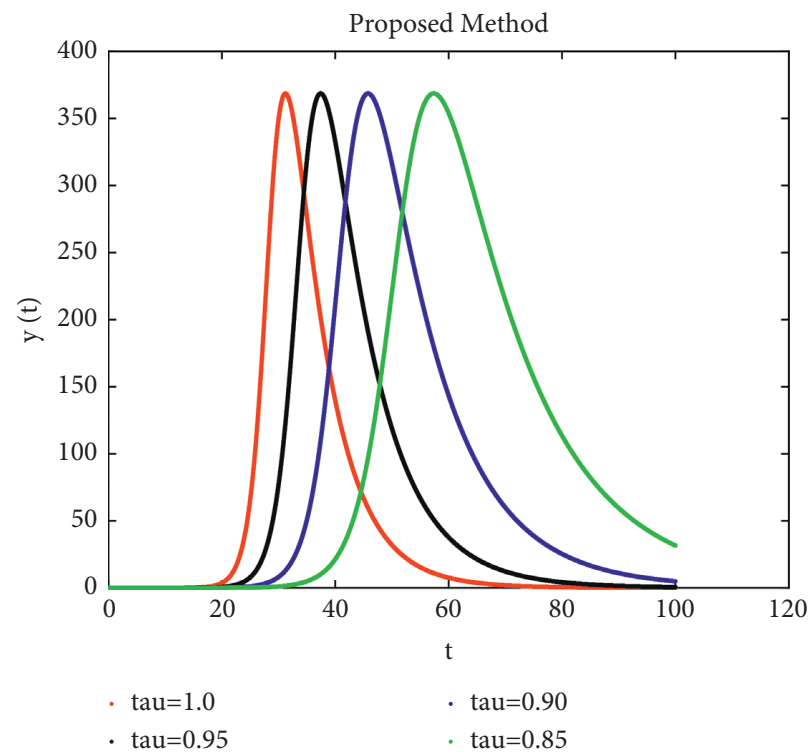

Figure 17: Dynamical behavior of infected cells at $\alpha=1$ and various fractal order $=0.85,0.9,0.95,1$ under Mittag-Leffler kernel.

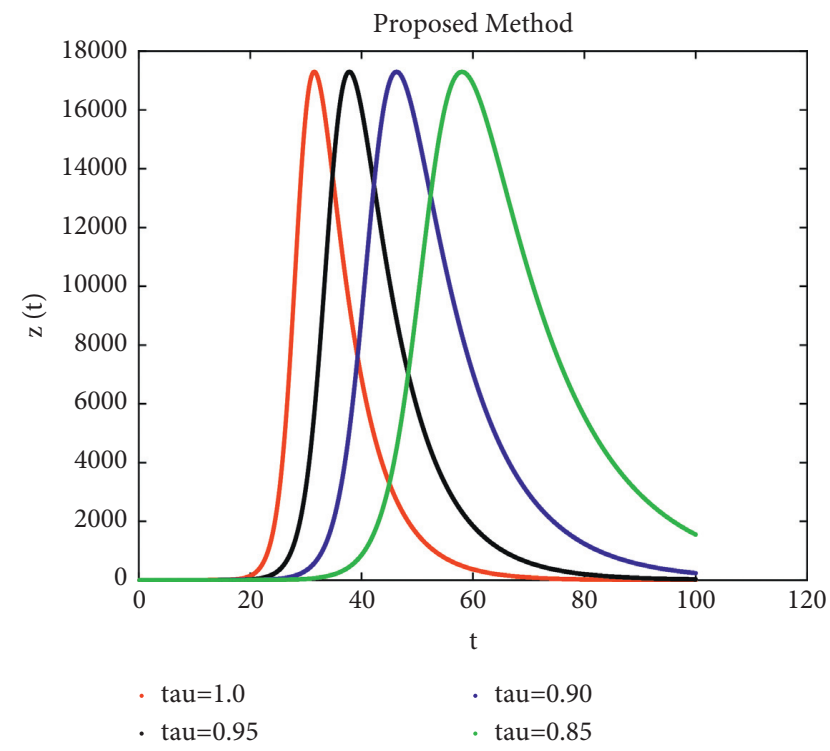

FIGURE 18: Dynamical behavior of free HIV virus particles at $\alpha=1$ and various fractal order $=0.85,0.9,0.95,1$ under Mittag-Leffler kernel.

\section{Conclusion}

In the current manuscript, we have applied the more generalized operators to study the relationship between HIV infection and its relation with CD4+ T-cells during primary infection. We have derived the results of the existence and uniqueness of the proposed fractal-fractional HIV model described by the operator in the Atangana-Baleanu sense. We have obtained numerical results through the Adams-Bashforth method. To visualize the dynamics of considered, we have simulated the obtained results through MATLAB-18. We have observed the impact of fractal dimension on the fractional order through graphs. The

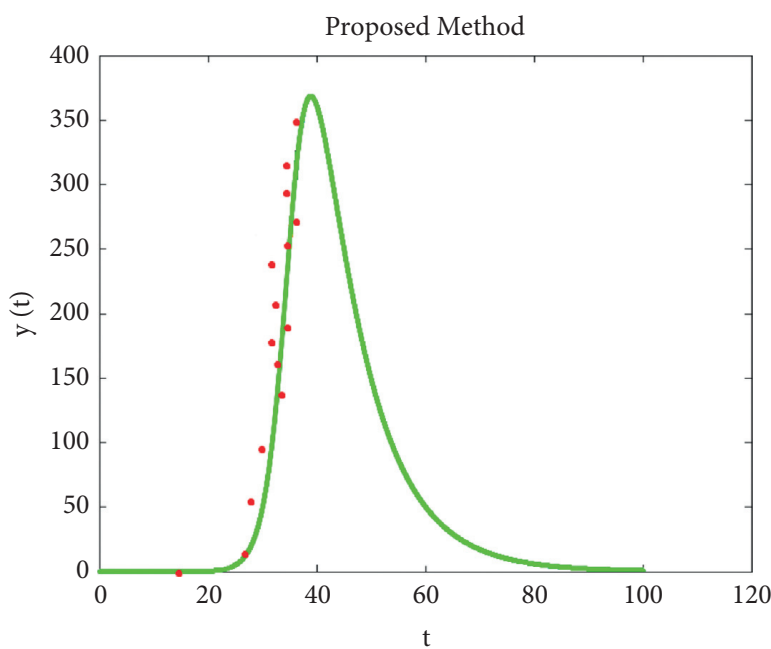

Figure 19: Comparison of simulated result with real data.

increase and decrease in fractal dimension affect the dynamics of the different compartments of the model. From the numerical simulations, we have observed that the fractalfractional idea produces better results in the sense of Atangana-Baleanu operators. So, we recommend that the Atangana-Baleanu fractal-fractional model gives the best results and may be more valuable to scientists and researchers. It is also observable from the numerical simulations that fractal-fractional order can capture more complexities than usual fractional derivatives. We keep hoping that this work may assist young researchers in a different direction of applied mathematics.

\section{Data Availability}

No data were used to support this study 


\section{Conflicts of Interest}

The authors declare that there are no conflicts of interest in this research study.

\section{Authors' Contributions}

All the authors contributed equally to this study.

\section{Acknowledgments}

The authors are grateful to the Basque Government (IT120719).

\section{References}

[1] A.-H. Abdel-Aty, M. M. A. Khater, H. Dutta, J. Bouslimi, and M. Omri, "Computational solutions of the HIV-1 infection of CD4+ T-cells fractional mathematical model that causes acquired immunodeficiency syndrome (AIDS) with the effect of antiviral drug therapy," Chaos, Solitons \& Fractals, vol. 139, Article ID 110092, 2020.

[2] M. M. A. Khater, A. E.-S. Ahmed, and M. A. El-Shorbagy, "Abundant stable computational solutions of AtanganaBaleanu fractional nonlinear HIV-1 infection of CD4+ T-cells of immunodeficiency syndrome," Results in Physics, vol. 22, Article ID 103890, 2021.

[3] L. Wang and M. Y. Li, "Mathematical analysis of the global dynamics of a model for HIV infection of CD4+ T cells," Mathematical Biosciences, vol. 20, no. 1, pp. 44-57, 2004.

[4] A. S. Perelson, "Modeling the interaction of the immune system with HIV," Lecture Notes in Biomathematics, vol. 83, pp. 350-370, 1989.

[5] A. S. Perelson, D. E. Kirschner, and R. D. Boer, "Dynamics of HIV infection of CD4+ T cells," Mathematical Biosciences, vol. 114 , no. 81, pp. 81-125, 1993.

[6] T. CHenry and Y. M. W. Frederic, "On the behavior of solutions in viral dynamical models," BioSystems, vol. 73, pp. 157-161, 2004.

[7] A. S. Perelson, A. U. Neumann, M. Markowitz, J. M. Leonard, and D. D. Ho, "HIV-1 dynamics in vivo: virion clearance rate, infected cell life-span, and viral generation time," Science, vol. 271, no. 5255, pp. 1582-1586, 1996.

[8] Y. Ding and H. Ye, "A fractional-order differential equation model of HIV infection of CD4+ T-cells," Mathematical and Computer Modelling, vol. 50, no. 3-4, pp. 386-392, 2009.

[9] G. Nazir, K. Shah, A. Debbouche, and R. A. Khan, "Study of HIV mathematical model under nonsingular kernel type derivative of fractional order," Chaos, Solitons \& Fractals, vol. 139, Article ID 110095, 2020.

[10] A. A. M. Arafa, S. Z. Rida, and M. Khalil, "Fractional modeling dynamics of HIV and CD4+ T-cells during primary infection," Nonlinear Biomedical Physics, vol. 6, no. 1, p. 7, 2012.

[11] S. Ahmad, A. Ullah, M. Arfan, and K. Shah, "On analysis of the fractional mathematical model of rotavirus epidemic with the effects of breastfeeding and vaccination under AtanganaBaleanu (AB) derivative," Chaos, Solitons \& Fractals, vol. 140, Article ID 110233, 2020.

[12] A. Ullah, T. Abdeljawad, S. Ahmad, and K. Shah, "Study of a fractional-order epidemic model of childhood diseases," Journal of Function Spaces, vol. 2020, Article ID 5895310, 8 pages, 2020.

[13] K. S. Nisar, S. Ahmad, A. Ullah, K. Shah, H. Alrabaiah, and M. Arfan, "Mathematical analysis of SIRD model of COVID-
19 with Caputo fractional derivative based on real data," Results in Physics, vol. 21, Article ID 103772, 2021.

[14] A. M. A. El-Sayed, H. M. Nour, W. E. Raslan, and E. S. ElShazly, "A study of projectile motion in a quadratic resistant medium via fractional differential transform method," $A p$ plied Mathematical Modelling, vol. 39, no. 10-11, pp. 28292835, 2015.

[15] S. Ahmad, A. Ullah, K. Shah, and A. Akgül, "Computational analysis of the third order dispersive fractional PDE under exponential-decay and Mittag-Leffler type kernels," Numerical Methods for Partial Differential Equations, vol. 16, p. 1, 2020.

[16] A. Atangana, "Fractal-fractional differentiation and integration: connecting fractal calculus and fractional calculus to predict complex system," Chaos, Solitons \& Fractals, vol. 102, no. 396, pp. 396-406, 2017.

[17] S. Ahmad, A. Ullah, T. Abdeljawad, A. Akgül, and N. Mlaiki, "Analysis of fractal-fractional model of tumor-immune interaction," Results in Physics, vol. 25, Article ID 104178, 2021.

[18] Fatmawati and M. A. Khan, "The dynamics of dengue infection through fractal-fractional operator with real statistical data," Alexandria Engineering Journal, vol. 60, no. 1, pp. 321-336, 2021.

[19] K. M. Owolabi, A. Atangana, and A. Akgül, "Modelling and analysis of fractal-fractional partial differential equations: application to reaction-diffusion model," Alexandria Engineering Journal, vol. 59, no. 4, pp. 2477-2490, 2020.

[20] S. Ahmad, A. Ullah, and A. Akgül, "Investigating the complex behaviour of multi-scroll chaotic system with Caputo fractalfractional operator," Chaos, Solitons \& Fractals, vol. 146, Article ID 110900, 2021.

[21] https://www.unaids.org/en/resources/fact-sheet. 